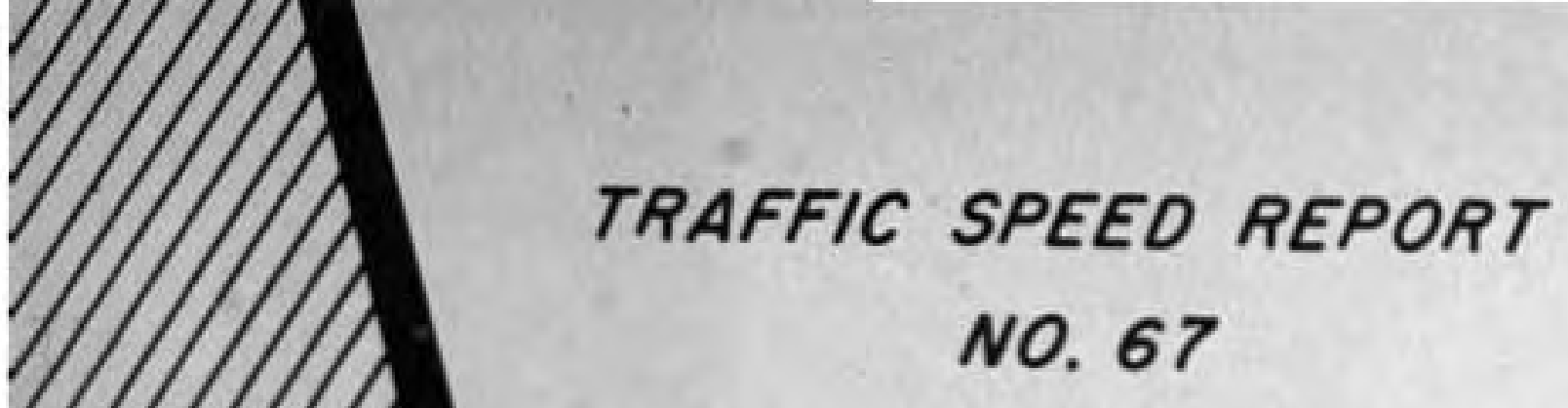

\title{
TRAFFIC SPEED REPORT NO. 67
}

NOVEMBER 1959

NO. 26

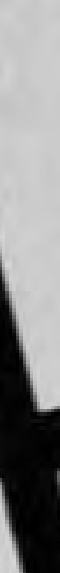

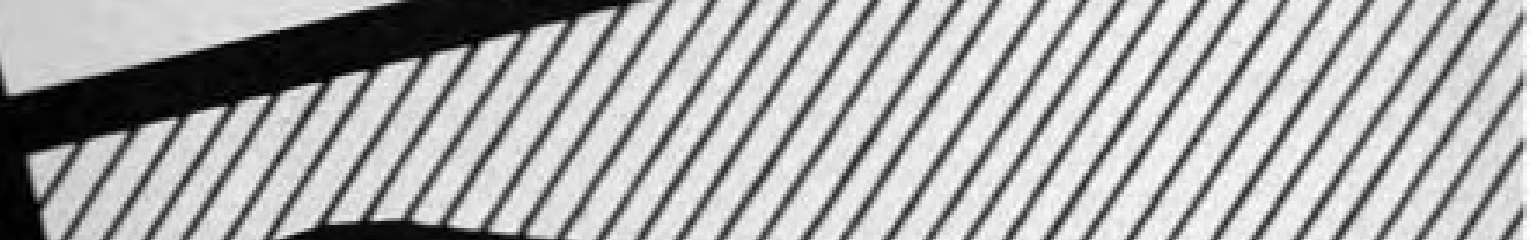

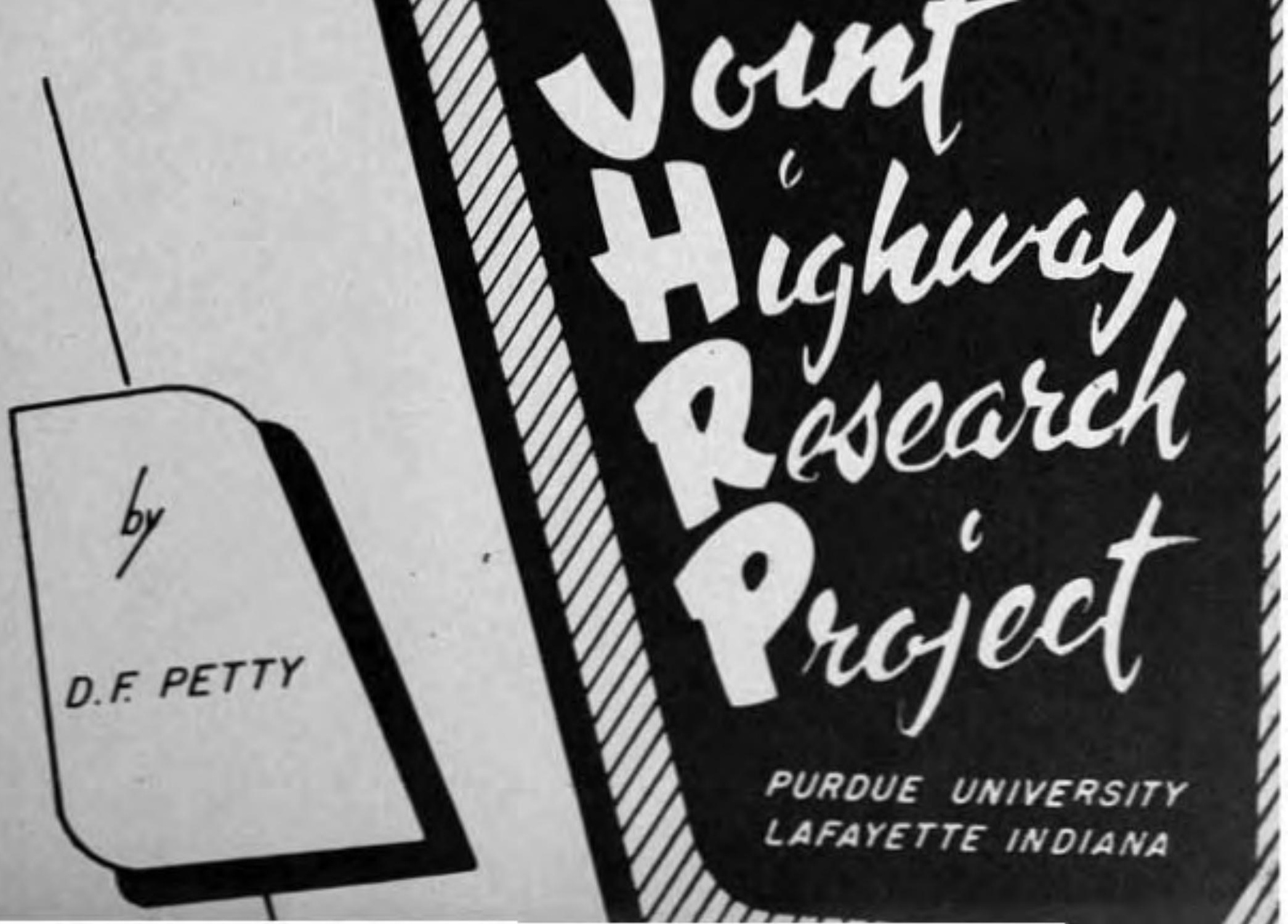


Progress Report

TaAFIC SPESD RSTORT 10, 67

To:

Frovt:
K. B. Hoods, Dirvetor

Joint Highnay Rosearch Froject

H. L. Michael, Assiotant Director

Joint Highay Resoerch Frofect
Hovenber 19, 1959

File: $8-3-3$

Project: $6-36-10 \mathrm{C}$

Attached is Traffle Speed Raport No. 67 which has bean prepared by Mr. Donald F. Petty, Research Assistant on our staff under the direction of Professor H. L. Michael.

This report prosents the speed dnta and an analyols of thesedats collocted during the sumar of 1959. The peoject has conducted aimilar studies at the sans locations seni-annualiy for the pat twenty years.

Of particular intoreat in tho rosulto of this study is the 6.2 NPH Increase in the average speod of heavy trucks on four-lnns hichuays. This increave is probably due to the 5 IIfH increase in the spoed linit for such trueks on such hifhays.

Copies of this roport vin be distributed as usual to the Indiana Stato Pollce, the Indiana Orfice of Irafrle Safoty and the Dureau of Public Roads. The report is submitted for the record.

HLMspg

Respectruly submitted,

2. 1. Tuellad

H. L. Whaol, Secretary

Attachmont

ea: F. L. Ashbaucher

J. 2. Coopar

W. L. Dolch

V. H. Coetz

F. F. Havoy

G. A. Havedins (H, B. Scott)

C. A. Leonards
J. F. Hotaughlin

R. D. 167os

R. B. 1811 s

C. B. Vocolgesang

J. L. Waling

J. B. HIson

B. J. Yoder 
Progress Rapout

TRAFFIC SPSBD REPOIT No, 67

Donald Fo Potty, Besoarch Assistant

Jolnt Hzghnsy Rasearah Project

Profect llo: Co-36-10C

Filo llo: 8-3-3

Purdus Univarsity

Lafayette, Indians

Novenber 19, 2959 
Digitized by the Internet Archive in 2011 with funding from

LYRASIS members and Sloan Foundation; Indiana Department of Transportation

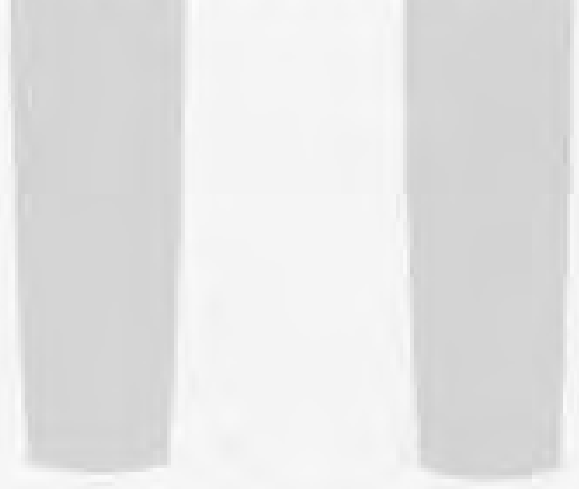




\section{IIST OF TABLES AWD FIGUIES}

TABIES

TABIS Nos

SUAKARY OF SPOT SPEBD OBSBRVATTOAS ............. I

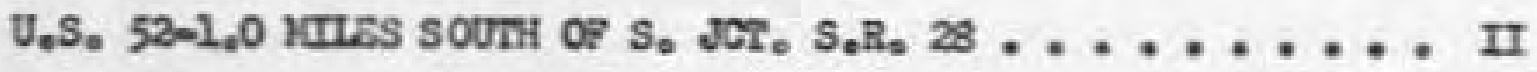

U.S. 52-1.0 ITIES WEST OP KIOUIDIFE ............. III

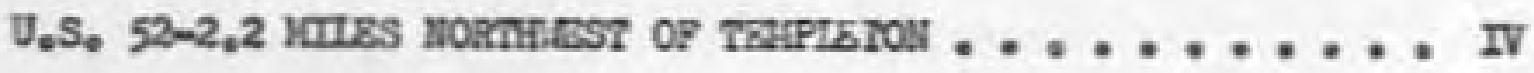

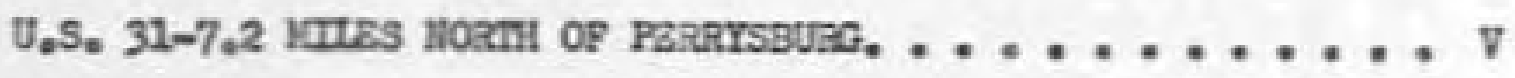

$S_{0} R_{0} 25-.7$ ITIES SOUTH OF ARBRTCUS, ............ VI

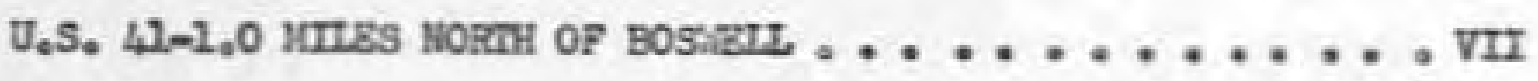

PIGURBS

EICUIS NOS

PSRCBNTIS SPESD CURVES

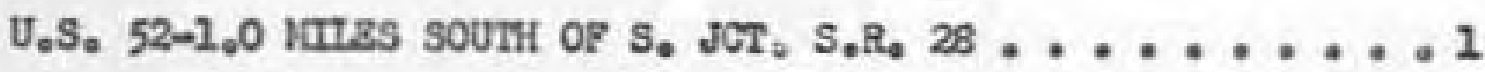

U.S. 52-1.0 1TIZS tTBST OP KLOHDIK ............. 2

U.S. 52-2.2 MUES HORTHIBST OF TAIFLZTON .......... 3

$\mathrm{U}_{.} \mathrm{S}_{.} 31-7.2$ IILES NORTH OF PERRYSBURG。 .......... 4

$\mathrm{S}_{0} \mathrm{R}_{0} 25-.7$ MIIES SOUTH OF ARBRTCUS, .......... 5

U.5. 41-1.0 MTLES NORTH OF BOSIRTL ............6

INDINM RURAL SPEED TRENDS 1942-1959......... 7

TREIDS IN PERCOTITS SFGBDS ATD SPEBD DIFFERATIAL

$1949-1959 \ldots \ldots \ldots$ 
Thls report covers spot speed observations made during July and August 1959. All observations were mede of free moving vohicles en lovel tangent sections of mural highways. The locations of the speod stations were the same as for provious studies and are as follows:

1. U.S. 52-1.0 miles South of South junction of $S_{0} R_{0} 28$ (Dual Lanes)

2. U.S. 52-1.0 milea Heat of Klondike (Dusl Lanes)

3. U.S. $52-2.2$ millos Northwest of Temploton (2 Iano)

4. U.S, 31-7.2 milas North of Perrygburg (2 Lano)

5. S.R. 25 $=0.7$ miles South of Amoricus (2 Lane)

6. U.S. 47-1.0 milles North of Boswoll (2 Lano)

An Electronatic Radar Speod Hoter was used to collect the data for this study. The meter was concealed as part of a rural mailbox and placed from two to three feet from the edge of the pavenent. It was directed along the highwey at a small angle with respect to the direction of traffic so that It was not necessary to make an angle correction to the readings.

The radar equipment used vill not operate properly if the voltage varies more than minus $1 / 2$ volt or plus 1 volt from the optimum twelve volte. The voltage vas therefore checked periodically in the field and maintained within the desired range. Also, correct calibration was mintained by periodically checking it with a $60 \mathrm{millo}$ per hour tuning fork.

The observers concealed thengelves fran traffic as nuch as local conditions permitted. A 150 foot cablo was used betwean the moter reading unit and the pick-up unit. It is therefore believed that the speeds of the observed vehicles were not influenced by the observers or the equipment. 
A sumasery of the results of tilis study, as well as of the last seven studies, is given in Table I. Indiana state law limits the speed of passenger cars and trucks under 5,000 pounds (GVit) to $65 \mathrm{millos}$ per hour. The speed limit of trucks over 5,000 pounds (GVil) is 50 miles per hour on all highways except that on four-lane hichways which have a modian strip of at least twenty feet In widh the speed limit is 55 millos per hour. This new speed linit for trucks has been in effect since about May 2959. However, very few speed limit sig: s have been posted in the state to reflect this change. It also is not known what percentage of the truck drivers know about $t$ is opeed limit change.

Truck spoeds are shoum in the tables for three groupst lifgt trucks, heavy trucks, and all trucks; also it is noted whether the road is 2 lane or 4. Lane. AIl 4 lane observation stations were on highways with a median of groater than twenty foot. Since weichts were not taken in this study, light tracks (less than 5,000 pounds) were considered to be only panel trucks and pick-up tiucks。 All other types of trucks were classified as heavy (over 5,000 pounds).

The passenger car data were also classified in three groupst Indians, out-of-state, and all passenger cars. The classification was deternined by observine the license plate on each passonger car after it had passed the station.

So that an easy comparison can be made between the results of the present study and the last previous study, the results frcul each speed station for both studies are tabulated in Tables II through VII. All speed observations for this study were taken at approximately the same locations as for the provious study. 
Avorage and 85th percentils speeds were couputed for each vehicle group from the samplo of vehicles taken at each study site and scme of the difforences between average speeds thus computed and average speeds simflasiy computed for the last study (December-larch 1959) a re briefly discussed. The average speed for all passenger cars increased $0.2 \mathrm{mlles}$ por hour since the last otudy while the average speed for all $t$ rucks increased 1.6 miles por hour. Indlana pasenger cars increased their average s peed on t wo-lane h1 phways by 0.1 miles per hour wh1le their average on four-lane h1 ghnays increased 0.2 miles per hour. Out-of-state passenger cars decreased on average speed by 2.4 milles per hour on two-lane highways tinile their average speed on four-lane highways decreased by 1.6 iniles per hour.

The a verage speed for 1 ight trucks decreased $0.7 \mathrm{mill}$.es per hour on $t$ woo lane highways and 3.6 milles per hour on four-lane highweys. Heavy trucks increased their a vorage spead on two-lane highways by 1.2 miles per hour and 6.1 milles per hour. on four-lane highways. This average speed increase for heavy trucks on fourlane highways is probsbly the effect of the new Indiana speed limit.

Trend information on average speeds and 85th percentile speeds for passenger cars and trucks is show in Table $I$ and Figures 7 and 8 。 
TABLE I

SUMKARY OF SPOT SPYSD OBSERVATIONS

ON IRDIANA HIGHAYS

(Free-Noving Vehicles on Lovel, Tangent Sections)

\begin{tabular}{|c|c|c|c|c|c|c|c|c|}
\hline \multirow{2}{*}{\multicolumn{2}{|c|}{$f$}} & \multicolumn{4}{|c|}{ Passenger Cars } & \multicolumn{3}{|c|}{ Trucks } \\
\hline & & $\begin{array}{l}\text { Ind } \\
\text { Mean }\end{array}$ & $\begin{array}{c}\text { Ion-Ind } \\
\text { Kean }\end{array}$ & $\begin{array}{l}\text { A13 } \\
\text { Mern }\end{array}$ & $\begin{array}{c}\text { A1] } \\
65 \text { par }\end{array}$ & $\begin{array}{l}\text { Light } \\
\text { Kean }\end{array}$ & $\begin{array}{l}\text { Heavy } \\
\text { Hean }\end{array}$ & $\begin{array}{l}\text { A21 } \\
\text { Kean }\end{array}$ \\
\hline \multirow[t]{7}{*}{$\begin{array}{l}\text { Two-Lane } \\
\text { Hightheys }\end{array}$} & Feb. 056 & 56.9 & 58.0 & 55.9 & 63,2 & 47,1 & 43.2 & 44.6 \\
\hline & Aug $_{0}: 56$ & 55.0 & 56.3 & 55.5 & 63.4 & 50,6 & 45.5 & 26.6 \\
\hline & Mex 157 & 55.6 & 59.1 & 56.2 & 64.0 & 50.2 & 44.8 & 46.1 \\
\hline & Ausg: 57 & 55.5 & 56.7 & 55.2 & 62.1 & 52.7 & 45.8 & 47.3 \\
\hline & Aug $_{0} 158$ & 54.3 & 56.3 & 55.0 & 62.7 & 53.0 & 46.3 & 47.8 \\
\hline & Mar. 159 & 55.5 & 57.7 & 56.1 & 63.9 & 50.4 & 45.4 & 46,3 \\
\hline & Auge $: 59$ & 55.6 & 55.3 & 55.9 & 63.2 & 49.7 & 46.7 & 48.0 \\
\hline \multirow[t]{7}{*}{$\begin{array}{l}\text { Four-lane } \\
\text { Highoreys }\end{array}$} & Fab.0 56 & 58.1 & 60,2 & 58.7 & $65 . ?$ & 47.8 & 45.2 & 4.5 .8 \\
\hline & Augo ${ }^{156}$ & 57.4 & 58.8 & 58,2 & 66.8 & 49,6 & 46.0 & 47.4 \\
\hline & Hay 157 & 59.9 & 63.6 & 61.0 & 69.0 & 52.2 & 46.0 & 47.9 \\
\hline & Aug: 157 & 57.5 & 59.9 & 58,5 & 64.8 & 52.0 & 46.6 & 47.6 \\
\hline & Aug $0_{0}: 58$ & 58.0 & 59.6 & 53.7 & 65.0 & $5 t_{t .} 3$ & 49.0 & 50.0 \\
\hline & Mar. 159 & 58.2 & 61.7 & 59.0 & 64.9 & 53.6 & 47.0 & $48-4$ \\
\hline & Aug. 159 & 58.4 & 60.2 & 59.1 & 64.5 & 50.0 & 53.1 & 49.5 \\
\hline \multirow[t]{7}{*}{$\begin{array}{l}\text { All } \\
\text { Highweys }\end{array}$} & Pob.:56 & 56.0 & 58.6 & 56.3 & 63.8 & 47.3 & 44.0 & 4.9 \\
\hline & Aug: 056 & 55.7 & 57.3 & 56.4 & 64.5 & 50.2 & 45.6 & 46.9 \\
\hline & May 157 & 57.2 & 60.3 & 58.3 & 66.0 & 50.9 & 45.2 & 46.6 \\
\hline & Aug. 157 & 56.2 & 58.3 & 56.9 & 63.2 & 52.8 & 46.2 & 47.4 \\
\hline & Augo:58 & 55.7 & 57.9 & 56,5 & 63.2 & 53.4 & 47.5 & 48.7 \\
\hline & Mar. ${ }^{159}$ & 56.6 & 59.0 & 57.2 & 63.1 & 52.5 & 45.9 & 47.0 \\
\hline & Aug $: 59$ & 56.5 & 58.4 & 57.4 & 63.5 & 50.6 & 47,8 & 48.6 \\
\hline
\end{tabular}




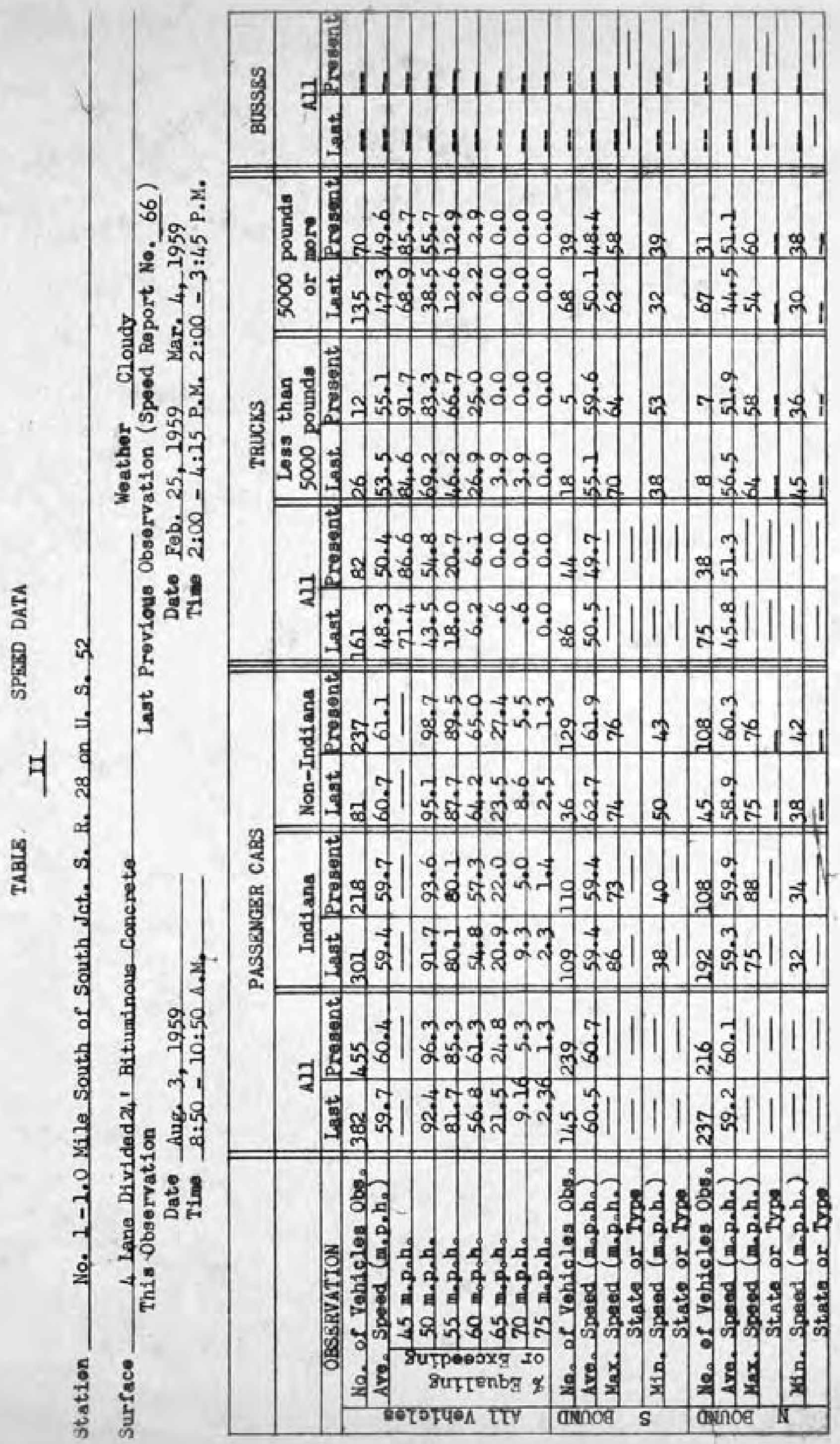




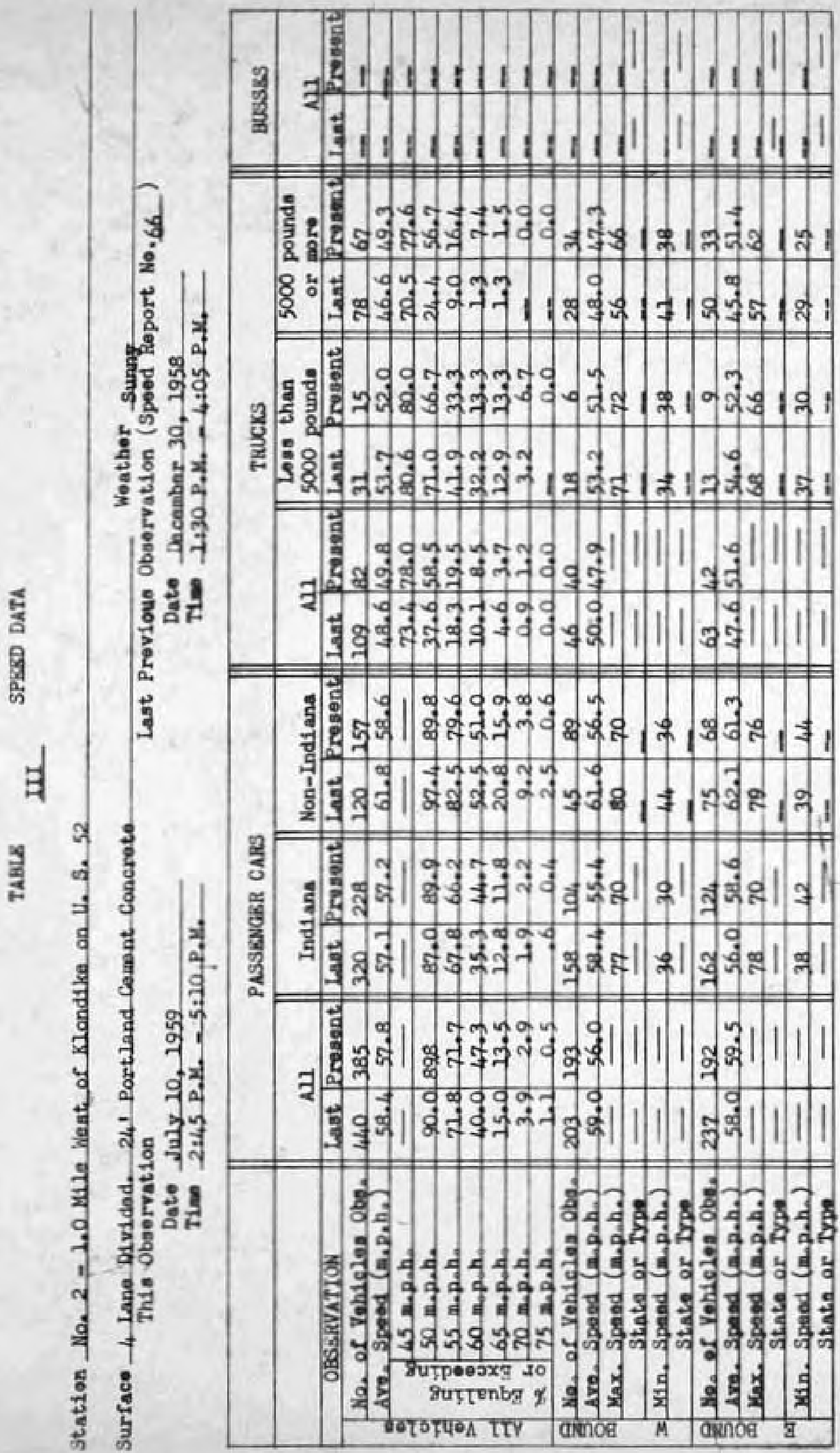




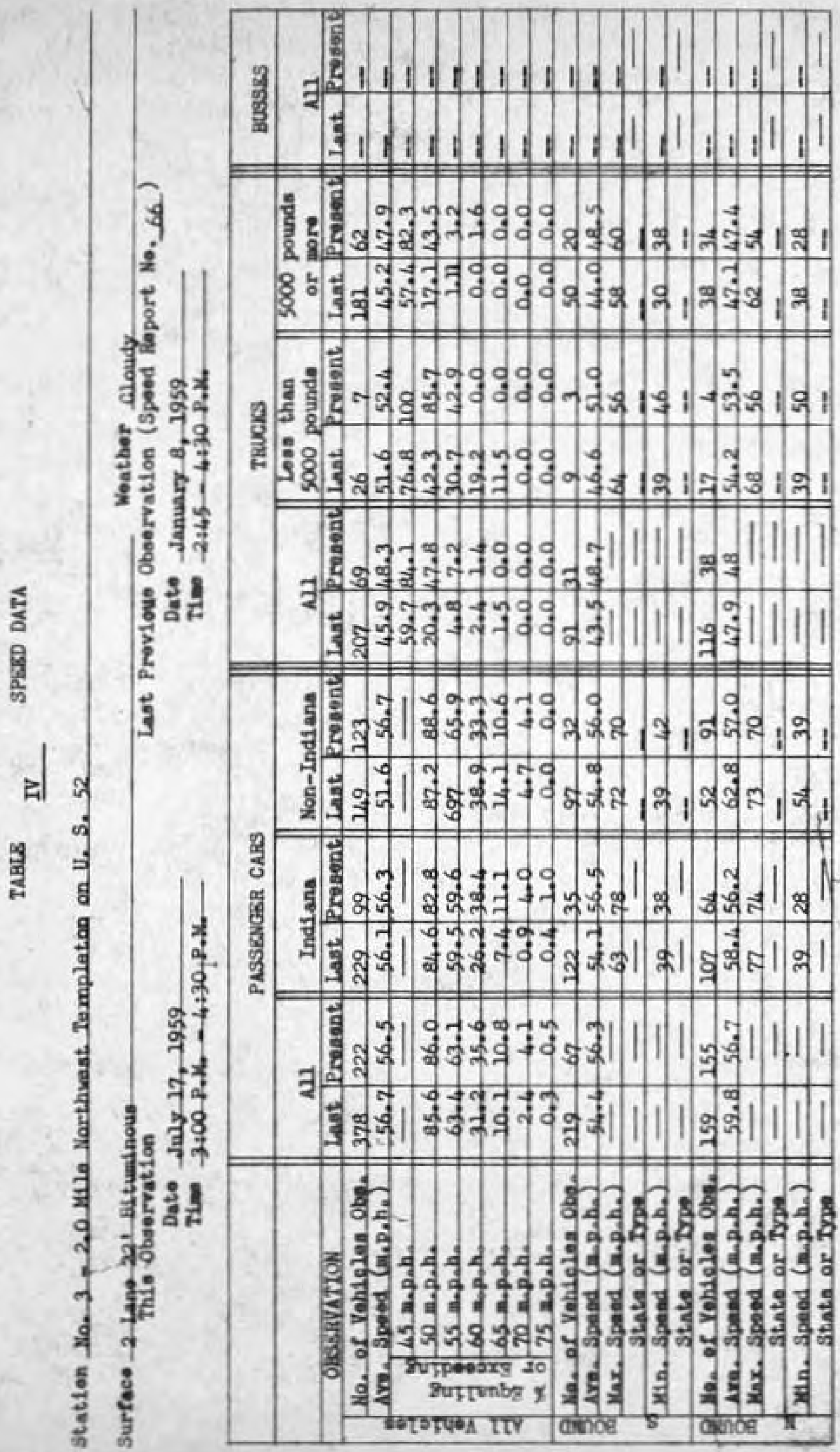




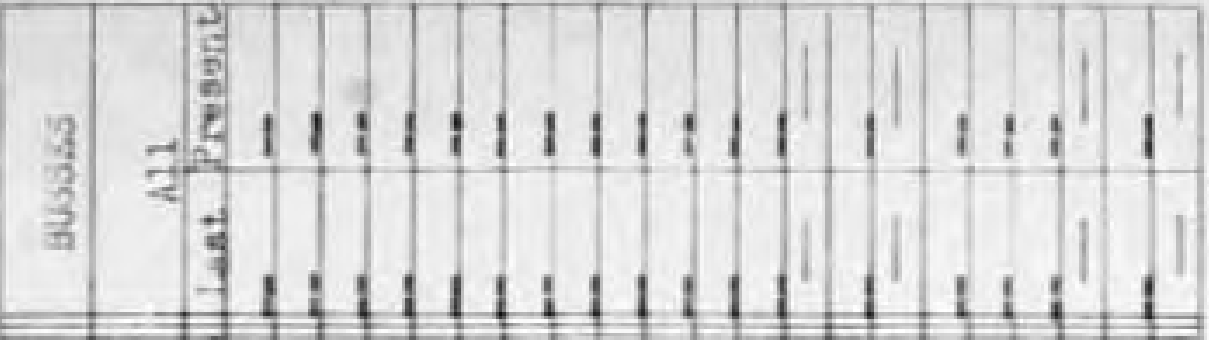

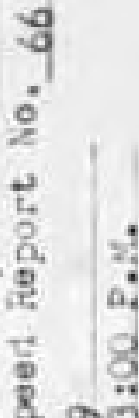

$\frac{8}{2}$,

L

:

İ⿴囗十

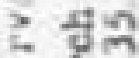

1 욜ㄹ

$\frac{5}{\frac{5}{3}}$

58

ํํำ

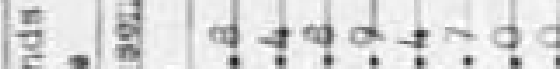

द 2 क्ष

2.

8ㅇํำ

थ

>

p.

2.

$\vec{n}$

द वै का

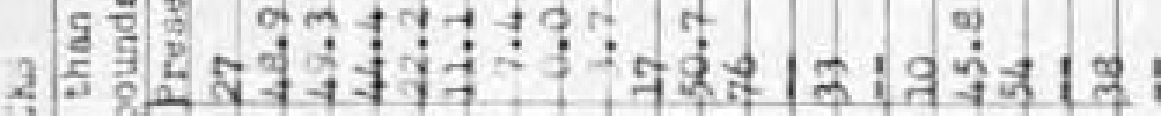

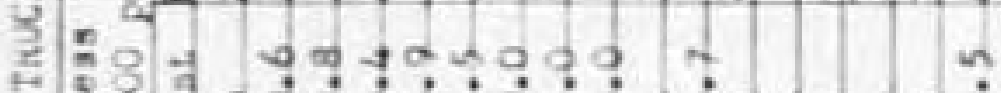

58

7.

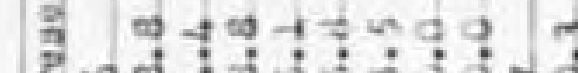

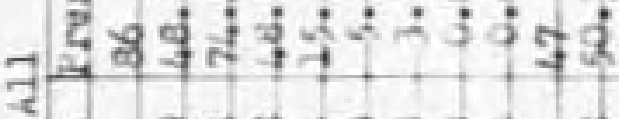

on:

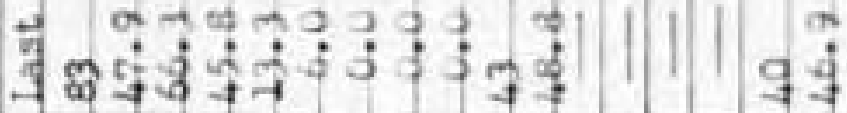

(4)

क E

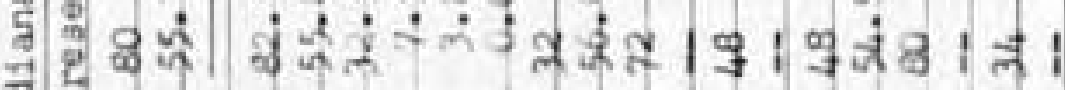

空

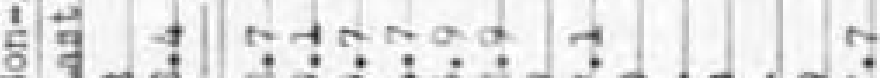

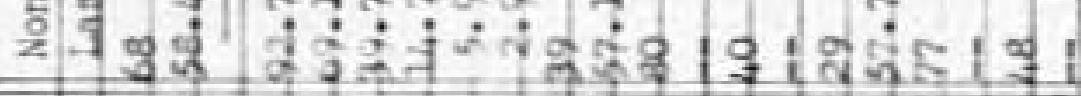

$\frac{9}{3}$

宩

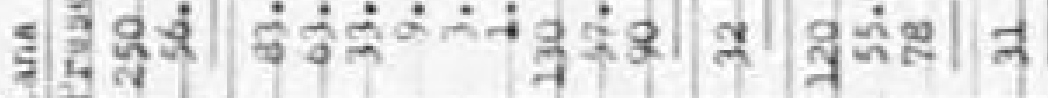

落

갰

点

3 은

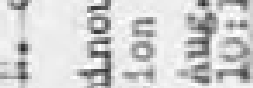

3 武? है:

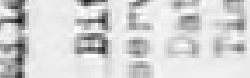

के

$-\frac{1}{2}$

is

当

द

औै

$+\frac{8}{3} \frac{1}{1}$

a

it

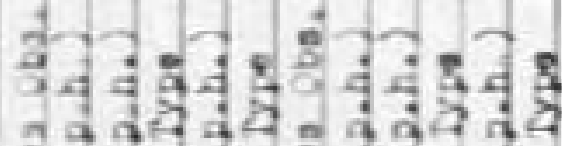

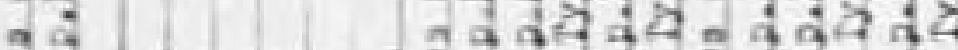

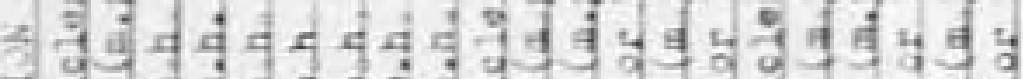

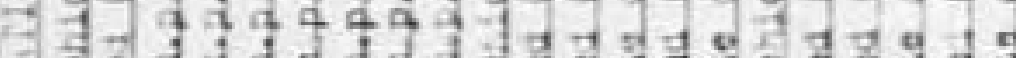

5 को

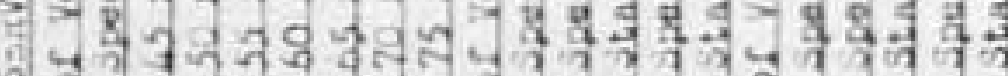

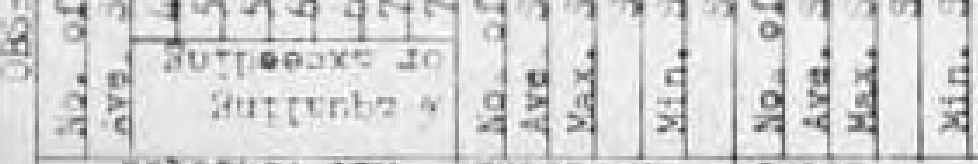

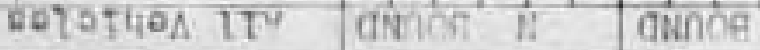




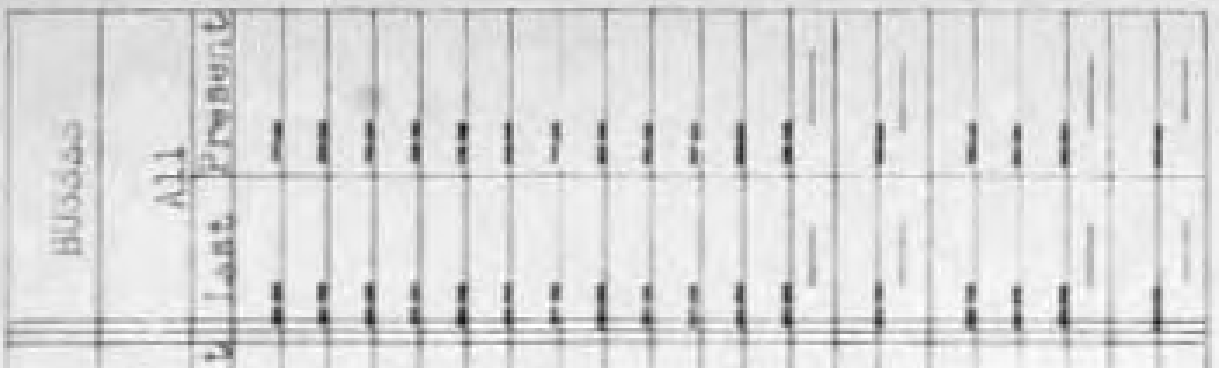

8

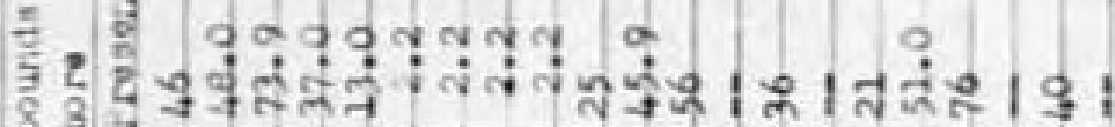

t)

$\rightarrow$

(1)

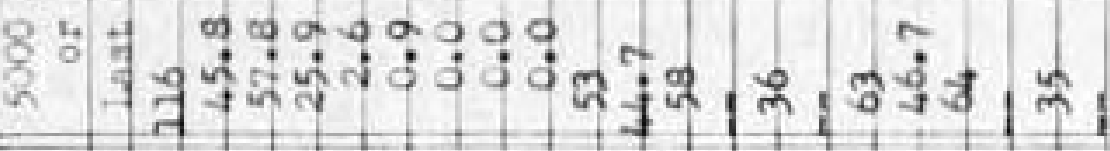

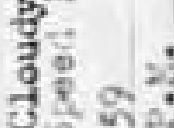

क्ष

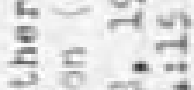

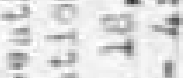

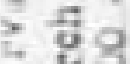

4.

兵

8

कूำ

3)

P.

$\overrightarrow{5}$

If

通

3.

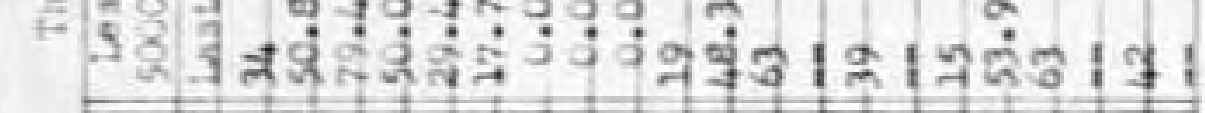

ने

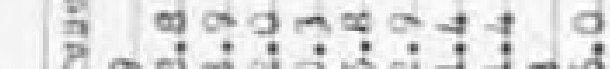

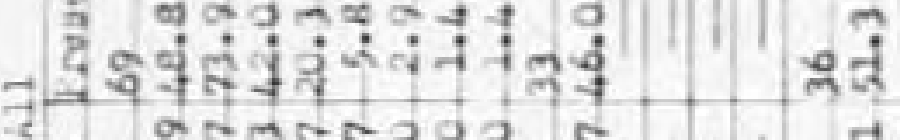

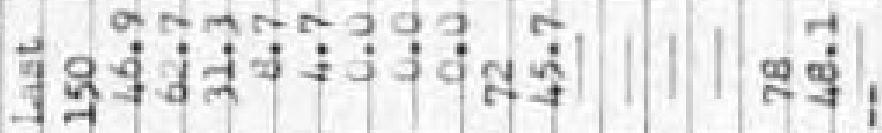

药

实

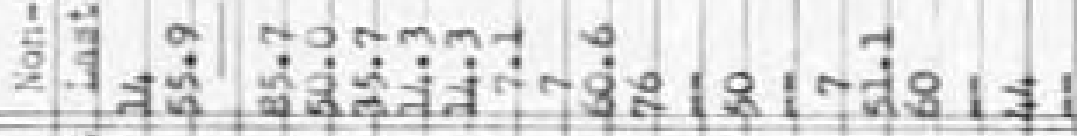

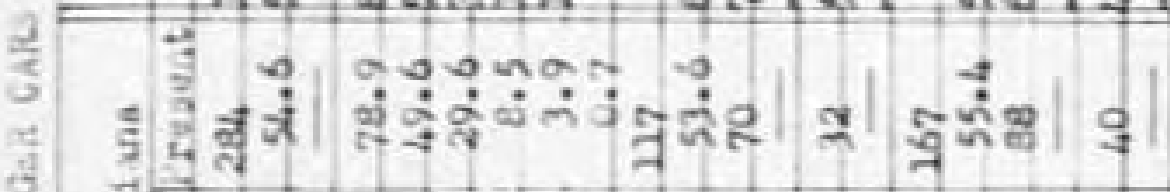

c.

है

c.

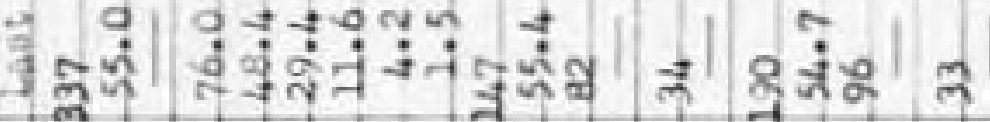

品

a

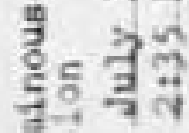

द

สี สิ สิ

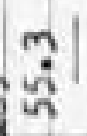

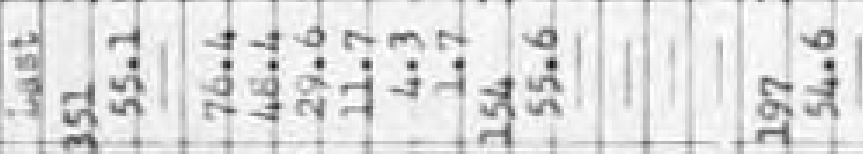

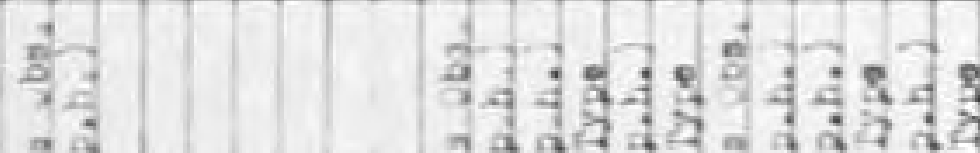

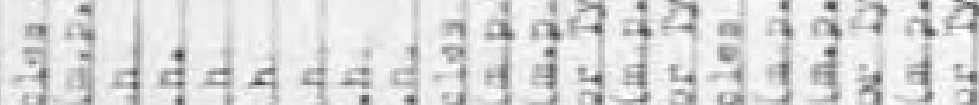

6.

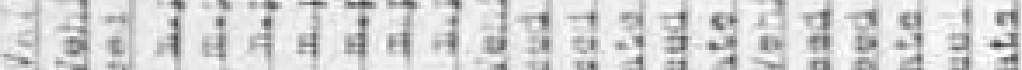

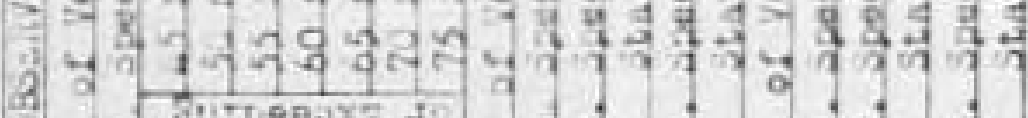

d.




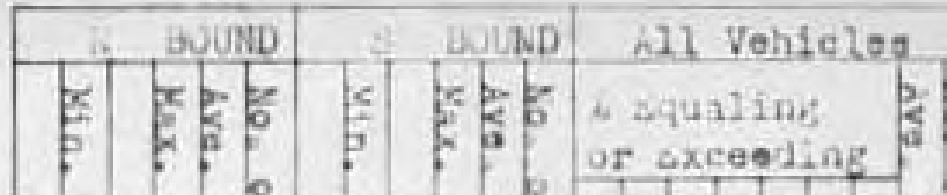

4

E

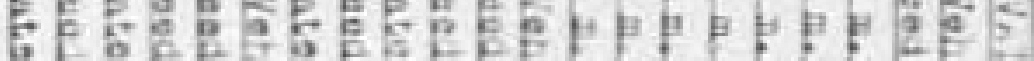

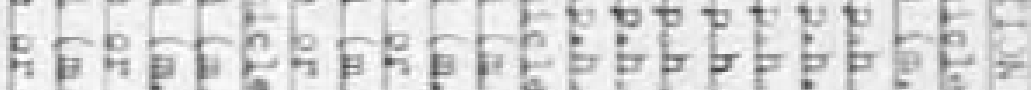

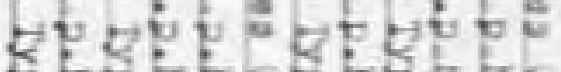

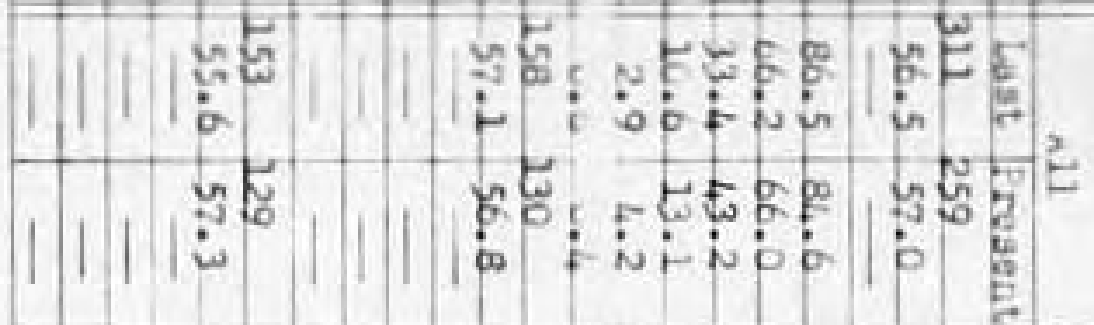

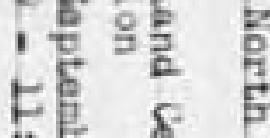

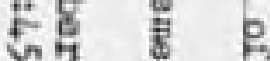

?

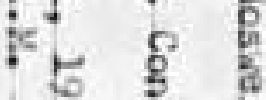

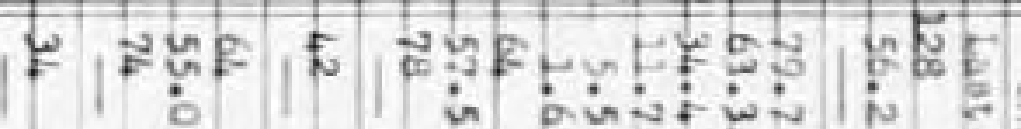

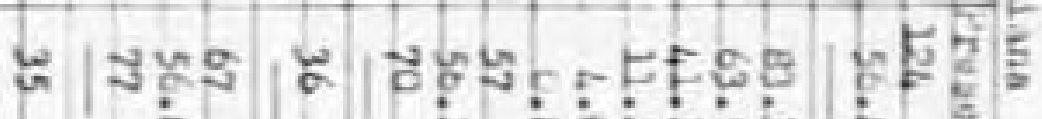

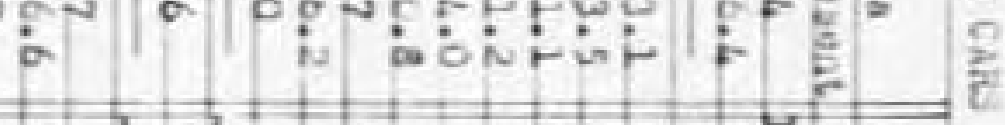
|

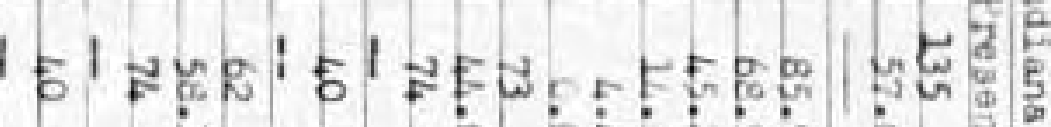

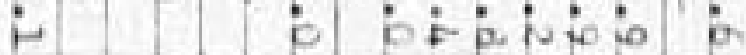

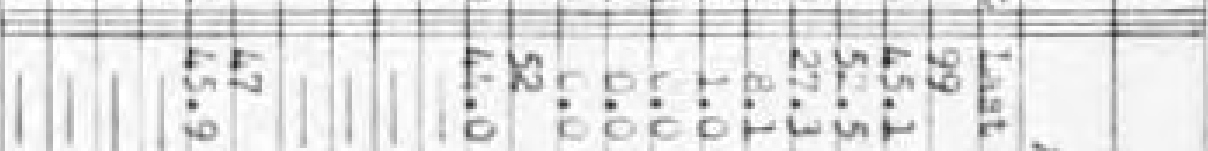

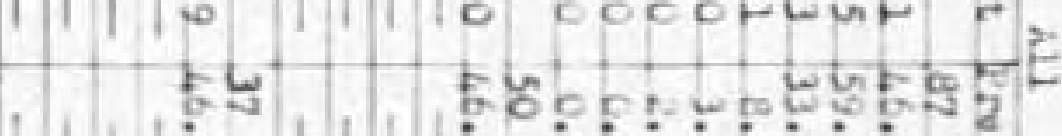

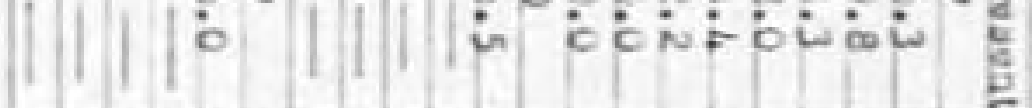

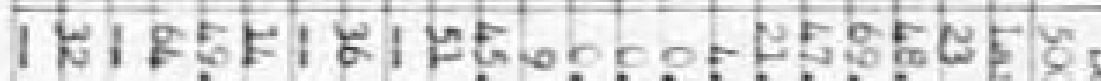
o. ${ }^{\circ}$ o.

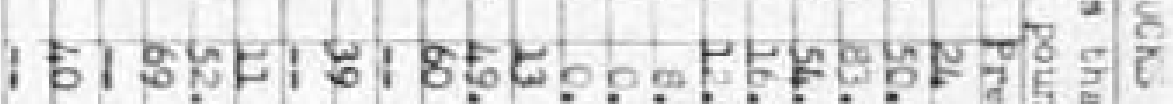

$$
\text { o in ócinisio } \frac{9}{4} 5
$$

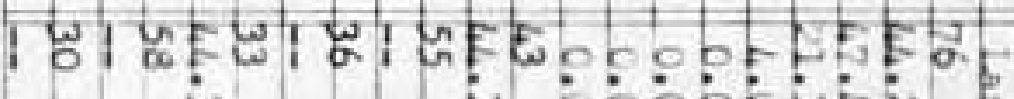

$$
\text { i }
$$

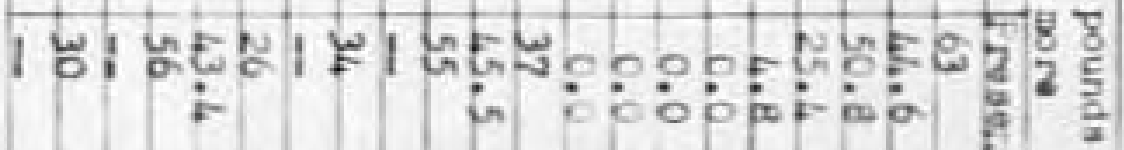

?

$\frac{1}{2}$

$150 \frac{9}{-2}$

50 u

岸点

te

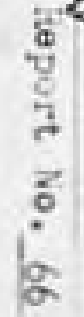




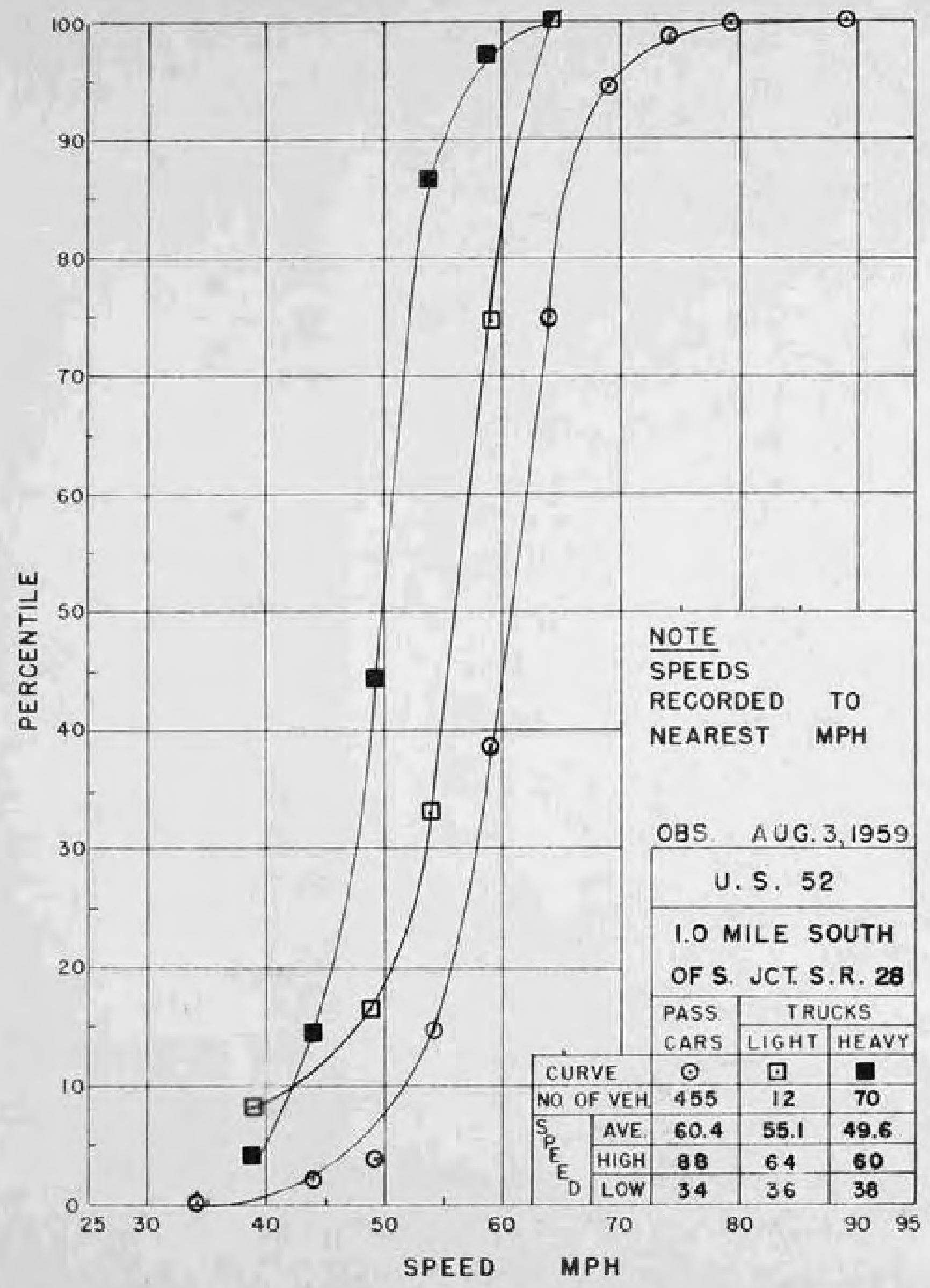

FIGURE I 


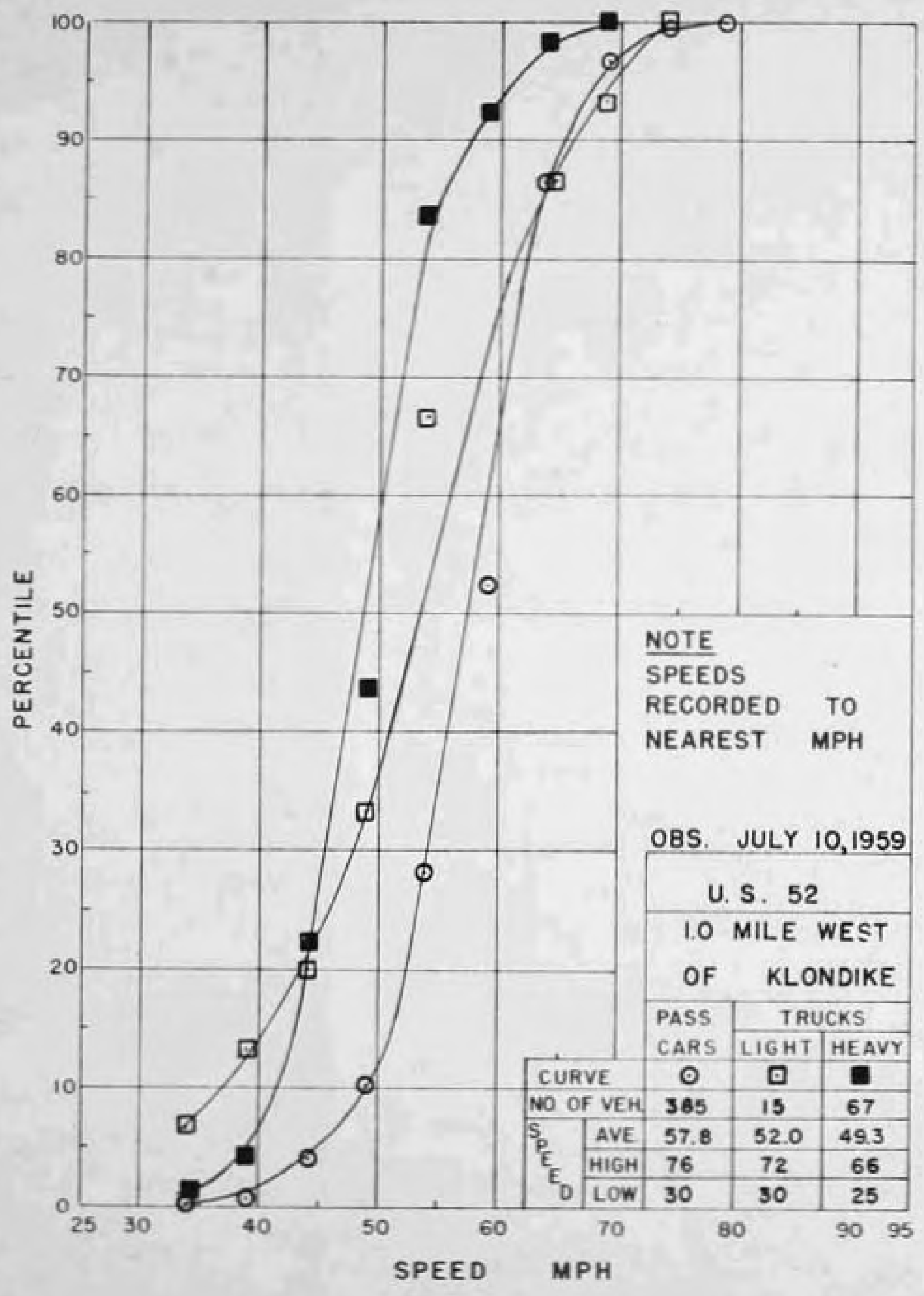

FIGURE 2 


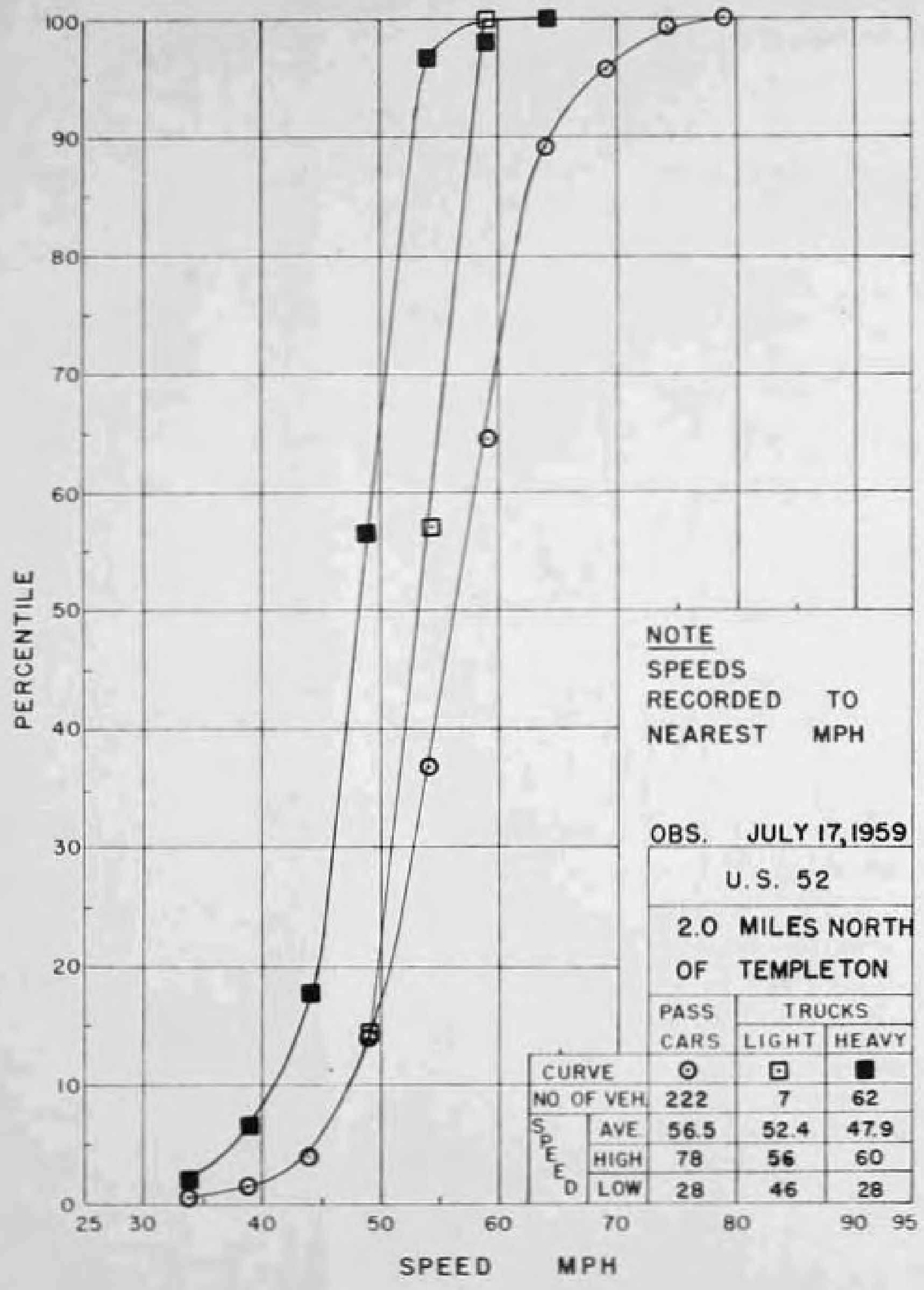

FIGURE 3 


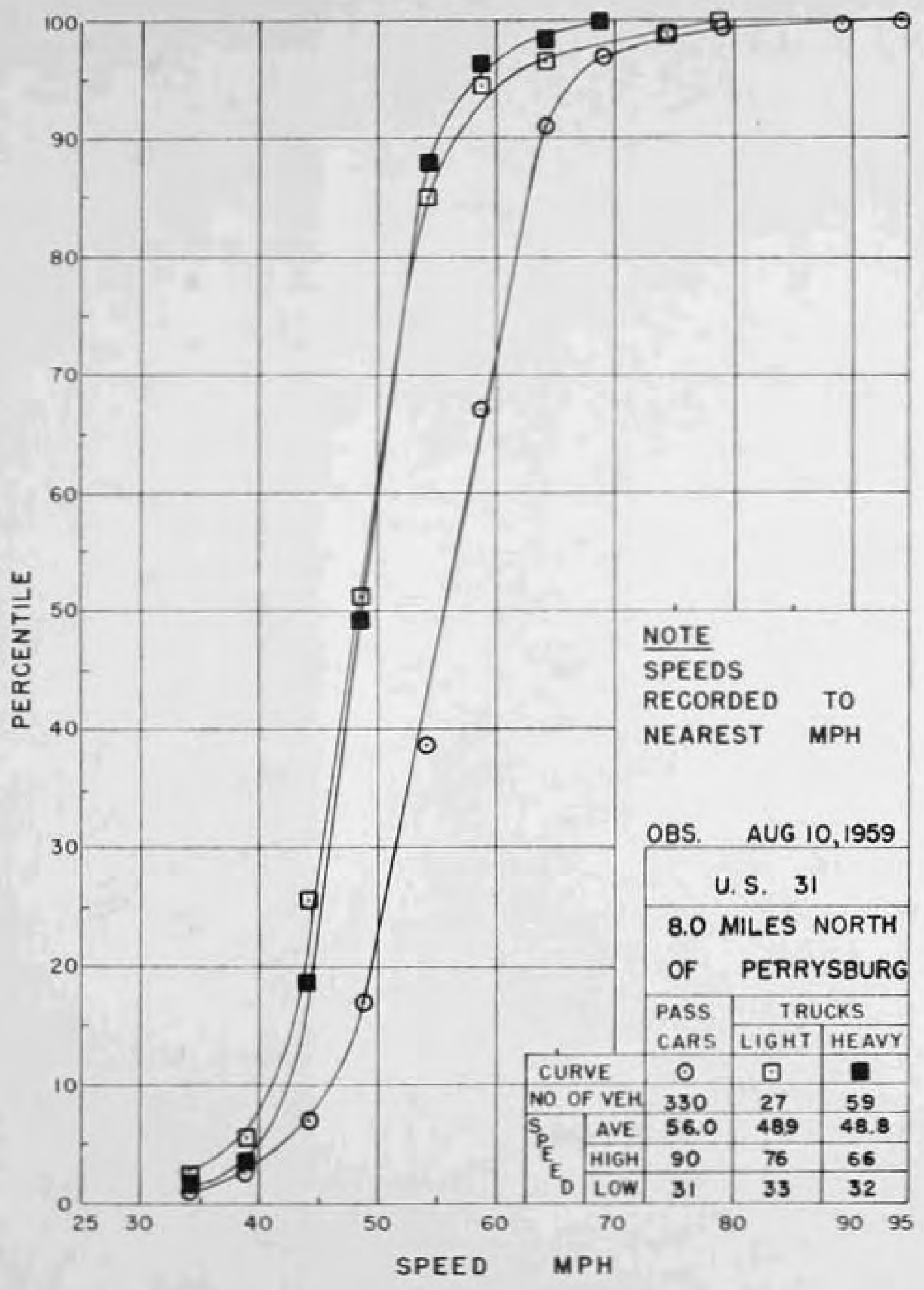




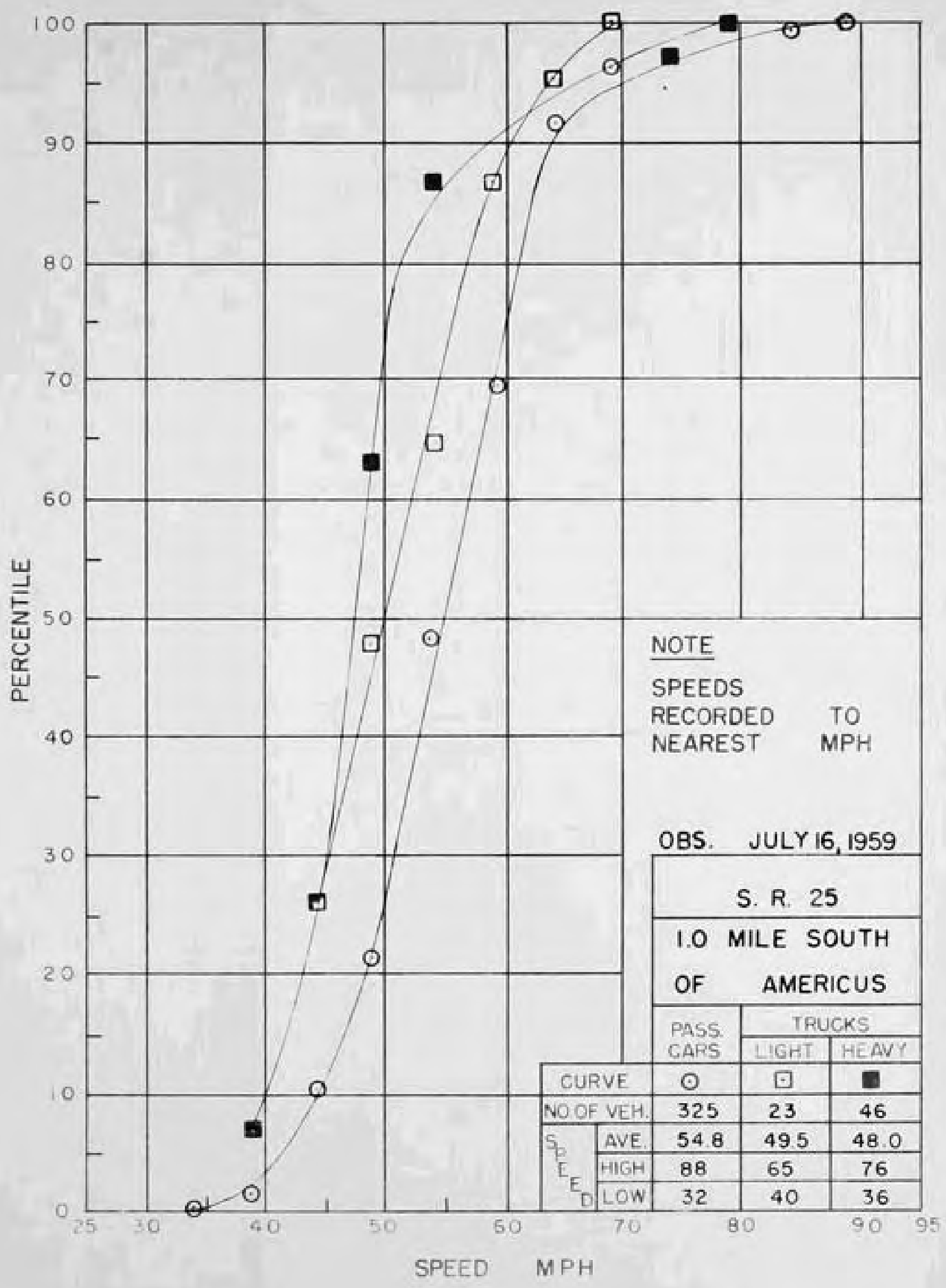




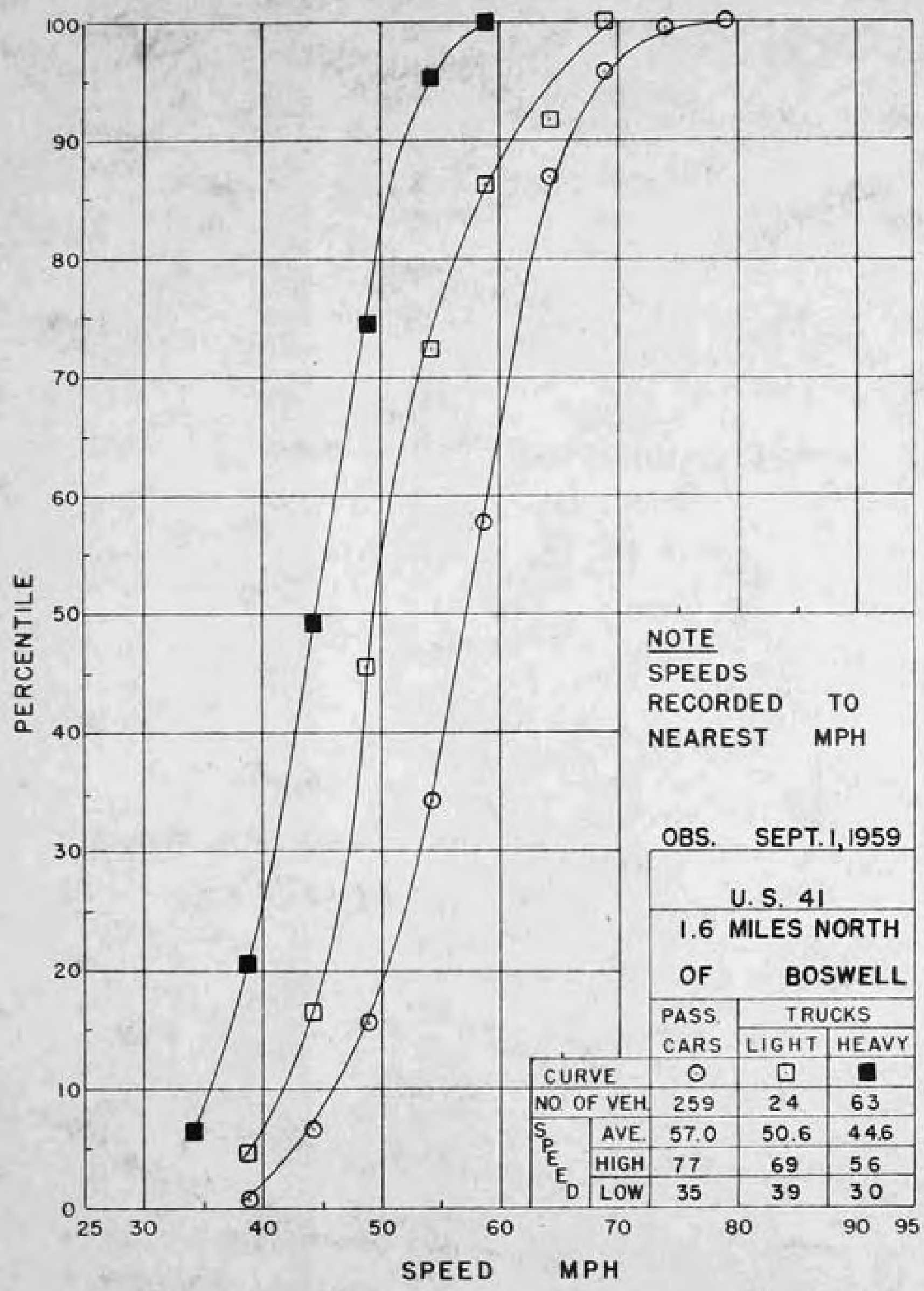

FIGURE 6 


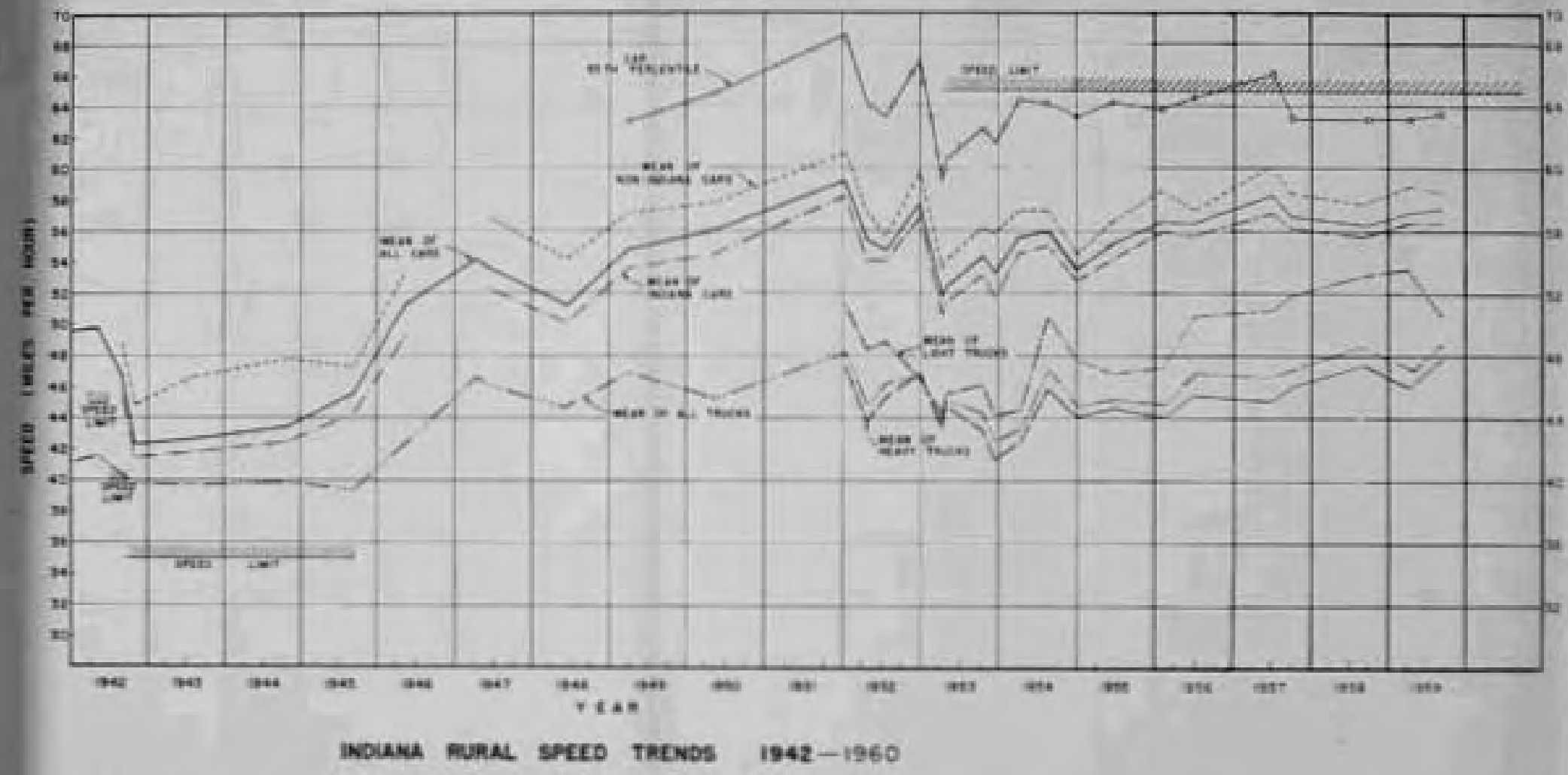

FIG 7 
